\title{
SNHG1 Long Noncoding RNA is Potentially Up-Regulated in Colorectal Adenocarcinoma
}

\author{
Niloofar Avazpour ${ }^{1}$, Mohammadreza Hajjari ${ }^{1 *}$, Seyed Reza Kazemi Nezhad ${ }^{1}$, \\ Maryam Tahmasebi Birgani ${ }^{2}$
}

\begin{abstract}
Colorectal cancer (CRC) is one of the most common types of cancer worldwide. However, the molecular mechanisms involved in CRC initiation and progression is remained to be unknown. It seems that lncRNAs, as the main and lengthy functional transcripts of the genome, have important roles in different cancers such as CRC. CRC-related lncRNAs are reported to be involved in diverse molecular processes such as metastasis, invasion, cell proliferation, and apoptosis. This study was aimed to analyse the expression level of lncRNA SNHG1 in colorectal adenocarcinoma and normal tissues. We performed an in silico analysis on a large cohort and confirmed the results by experimental analysis of clinical samples through real-time PCR. Our findings demonstrated that that SNHG1 is potentially overexpressed in tumor tissues compared with adjacent normal tissues. The expression level of $S N H G 1$ was shown to be potentially associated with clinicopathological features of tumors. The current study suggests the potential role of SNHG1 in colon cancer progression.
\end{abstract}

Keywords: SNHG1- RNA- long noncoding- gene expression- cancer- colorectal

Asian Pac J Cancer Prev, 21 (4), 897-901

\section{Introduction}

Colorectal cancer (CRC) is one of the leading causes of cancer death in men and women totally. In spite of decline in incidence and improvement, there is an emergent need to early detection and treatment of CRC (Siegel et al., 2017; Torre et al., 2012). In Colorectal cancer, as a multi-step and multifactorial disease, it has been shown that gene expression plays a pivotal role in tumor progression (Birkenkamp-Demtroder et al., 2002).

Recent studies have shown that up to $90 \%$ of the genomic DNA are transcribed to a complicated network of transcripts. The major transcripts which are over 200 nt in length includes Long Non-coding RNAs (LncRNA) (Ponting et al., 2009). Also, it has been revealed that lncRNAs, as the major regulators of gene transcriptional system, are involved in the different biological process such as cell cycle regulation and cytoplasmic transport (Wang et al., 2011).

Much investigations have been focused on characterizing the role of IncRNAs in the prognosis of CRC (Han et al., 2015; Xie et al., 2016; Xu et al., 2014). For example Kogo et al., (2011) indicated that HOTAIR, as modulator of the cancer epigenome, promotes metastasis, recurrence, and prognosis of CRC.

In this study, we studied the expression level of SNHG1 lncRNA (small nucleolar RNA host gene 1). Its gene is localized at 11q12.3 and has 11 exons. Aberrant expression of this lncRNA has been reported in some cancers such as non-small cell lung cancer (You et al., 2014), glioma (Wang et al., 2017), Lung squamous cell carcinoma (Zhang et al., 2017), neuroblastoma (Sahu et al., 2016) and etc. It seems that this lncRNA can function both in Cis and in trans to regulate transcription, promoting tumorigenesis and cancer progression (Sun et al., 2017). However, the role and mechanism of SNHG1 in colorectal adenocarcinoma have not been completely understood.

In order to determine the potential clinical relevance of SNHG1 in colorectal adenocarcinoma, we analyzed the expression pattern and dysregulation of this RNA by utilizing RNA-seq data and clinical information from a significant number of colorectal adenocarcinoma patients in The Cancer Genome Atlas (TCGA) and GEO datasets. The clinical validation demonstrated the potential role of SNHG1 lncRNA in colon cancer. The data suggested that $S N H G 1$ could be a potential biomarker and potential therapeutic target for CRC.

\section{Materials and Methods}

In silico expression analysis of SNHG1 gene in large cohorts

In the current study, we considered differentially expression of SNHG1 lncRNA using TCGA data from 
the Cancer Genome Browser database (https://genomecancer.ucsc.edu/) in order to determine whether $S N H G 1$ lncRNA was associated with the progression of CRC. To evaluate this, we performed this type of analysis using two datasets including TCGA colon and rectum adenocarcinoma (COAD-READ) gene expression data assayed by RNA-seq (Illumina Hiseq). The expression level of $S N H G 1$ was compared between colorectal adenocarcinoma tissues $(\mathrm{n}=380)$ and adjacent normal tissues $(\mathrm{n}=50)$. Also, the Nexus expression database was checked for further analysis of SNHG1 in colorectal tumors. Also, Kaplan-Meier survival analysis was used to explore the prognostic value of $S N H G 1$ through the TCGA database. The result shows that there was no significant difference in survival of CRC patients with higher and lower SNHG1 expression. (P-value=0.9023) (Figure 4).

\section{Validation study by clinical samples}

To support the in silico analysis, we examined SNHG1 expression level CRC tissues by qRT-PCR.

\section{Clinical samples}

A total of 40 samples comprising 20 colorectal adenoccarcinoma tumors and their normal 20 adjacent adenocarcinoma normal tissues were obtained from Iran National Tumor Bank. All of the tissue specimens were frozen in liquid nitrogen after collection and then stored in $-80^{\circ} \mathrm{C}$ until the extraction of Total RNA. The clinical and pathological parameters of these samples which were confirmed by pathologists are given in Table 1 . The process of the study was approved by Shahid Chamran University of Ahvaz, Iran.

\section{RNA Isolation and cDNA synthesis}

Total RNA was extracted from tissue samples using the $\mathrm{RNX}^{\mathrm{TM}}$-plus reagent (Cinnagen, Iran) following manufacturer's instructions. The quality and quantity of extracted RNA were checked by gel electrophoresis and spectrophotometry respectively. The cDNA was generated using the reverse transcription Kit (Takara, Japan) according to the manufacturer's instructions. One microgram of Total RNA in each sample was reversely transcribed into cDNA by Reverse transcription Kit (Takara, Japan) in a total volume of $10 \mu \mathrm{l}$ according to the manufacturer's protocols.

\section{Real-time quantitative PCR}

Real-time PCR analysis was performed in order to determine the relative expression level of target genes with the SYBR Premix ExTaq Kit (Takara, Japan) by the ABI Prism7500 system (Applied Biosystems, Foster City, CA, USA). All reactions were run in duplicate using SNHG1 specific primer as target gene and GAPDH as the internal control to normalize the RNA levels for different sample. The reaction mixture consisted of cDNA $(1 \mu 1), 5$ $\mu \mathrm{l}$ of $2 X$ SYBR Green I master mix (Takara, Japan) and $200 \mathrm{nM}$ of each primer in a $10 \mu \mathrm{l}$ reaction according to the manufacturer's instructions. The cycling conditions included an initial denaturation at $95^{\circ} \mathrm{C}$ for 30 s followed by 40 cycles of denaturation at $95^{\circ} \mathrm{C}$ for $5 \mathrm{sec}$, annealing/ extension at $60^{\circ} \mathrm{C}$ for $34 \mathrm{~s}$. The specificity of the PCR products was examined by melting curve analysis, followed by gel electrophoresis. The Livak method $(\Delta \Delta \mathrm{CT})$ was used to analyze differential gene expression (Livak et al., 2001). Serially diluted cDNA was used to obtain a standard curve for each gene transcript. The primers sequence were as follows: for SNHG1 Forward: 5' - ACGTTGGAACCGAAGAGAGC -3' and Reverse: 5'- GCAGCTGAATTCCCCAGGAT -3' and for GAPDH Forward: 5' - GTGAACCATGAGAAGTATGA-3' Reverse: CATGAGTCCTTCCACGATAC-5' . The sequence of the $S N H G 1$ PCR product was confirmed by Sanger sequencing.

\section{Statistical analysis}

For in silico analysis of SNHG1 expression in TCGA derived tissues, the statistical analysis was done by t-test through R-software integrated in cancer genome browser. the clinical study, Statistical analysis was done using the GraphPad Prism v6 software. Differences in RNA levels between the normal and tumor tissues of patients groups was assessed by paired Student's t-test. A P value $<0.05$ was considered as significant.

\section{Results}

SNHG1 is up-regulated in TCGA colorectal tumor tissues compared to normal tissues

Normalized expression ( $\mathrm{Z}$ score) derived from TCGA tissues of cancer genome browser was compared between groups of study. In TCGA (The Cancer Genome Atlas) data which applies RNA sequencing of large cohorts, we found that SNHG1 is significantly up-regulated in CRC tissues compared with adjacent normal tissues (Figure 1).

Experimental study of SNHG1 expression in colorectal adenocarcinoma tumor and adjacent non-cancer tissues

We further examined SNHG1 expression level in 40 tissues including 20 pairs of human CRC tissues and normal adjacent samples in order to investigate the

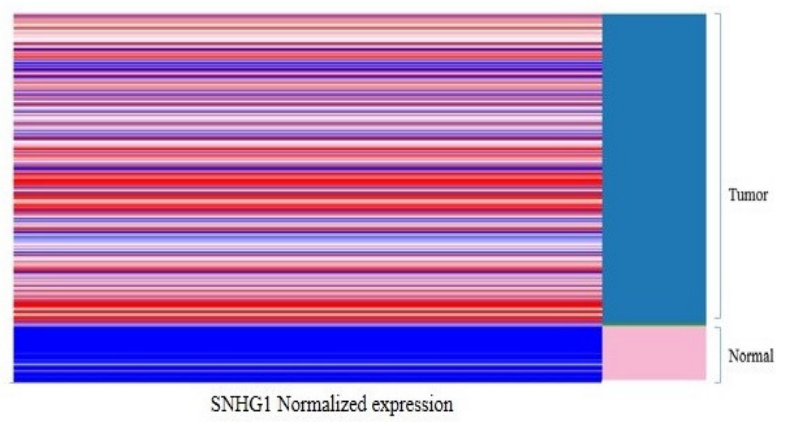

Figure 1. Heatmap Shows SNHG1 Expression Profile in TCGA Database. TCGA colon and rectum adenocarcinoma (COADREAD) gene expression data grouped in primary tumors (380) and normal solid tissues (50) and represents a heatmap .The data heatmap shows up-regulation in Colorectal cancer tissues (shown a red subgroup in the column) in comparison to normal tissues (shown a green subgroup the right column). The normalized gene expression data assayed by RNA-seq (Illumina Hiseq) has been shown in red $(Z>0)$ and blue color $(Z<0)$ in this heatmap. Data is derived from the Cancer genome browser database. 
Table 1. Clinical Characteristics of Colorectal Cancer Patients

\begin{tabular}{lc}
\hline Clinical parameters & Numbers \\
\hline Samples & 20 \\
Gender & 10 \\
$\quad$ Male & 10 \\
$\quad$ Female & \\
Age & 11 \\
$\quad<64$ & 9 \\
$\geq 64$ & \\
Tumor size & 10 \\
$\quad<5$ & 10 \\
$\quad \geq 5$ & \\
Tumor site & 12 \\
Colon & 8 \\
Rectum & \\
Grade of tumor & 19 \\
Low Grade & 1 \\
High Grade & \\
TNM stage & \\
Stage1-2 & \\
Stage3-4 & \\
Lymphatic invasion & \\
Yes & \\
No & \\
Weight Loss & \\
No & \\
\hline
\end{tabular}

TNM, Tumor-Node-Metastasis

potential roles of $S N H G 1$ in human CRC development. As shown in the Figure 2, $S N H G 1$ was up-regulated in tumor tissues compared to their match normal tissues (P-Value $<0.0064)$. The results potentially validated the results derived from our in silico analysis through cancer genome browser.

Correlation between SNHG1 expressions and patient's clinicopathological characteristics

We examined the correlation between SNHG1 expression and clinicopathological characteristics in CRC samples to find out whether $S N H G 1$ was associated

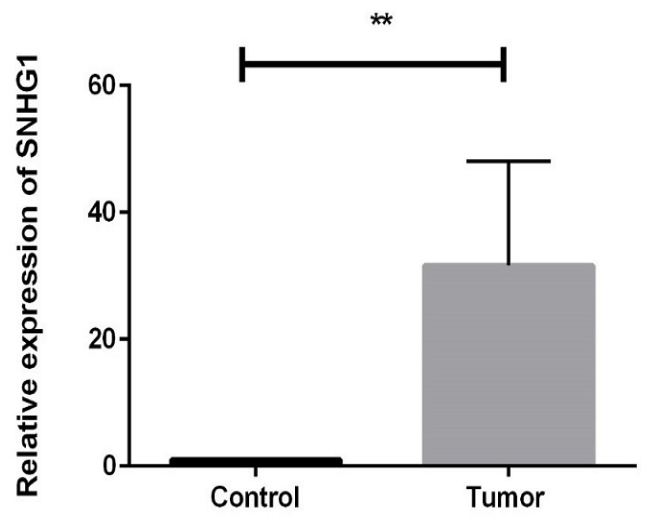

Figure 2. SNHG1 Expression Analysis in Colorectal Cancer Tissues and Corresponding Adjacent Normal Tissues. SNHG1 Real time-PCR expression data normalized to $G A P D H$ expression as internal control. Paired t-test result showed the higher an internal of the SNHG1 in colorectal cancer a higher tissues (P-value $=0.0064)$. reported Data are presented as mean \pm SEM.

with the progression of CRC. Our analysis showed up-regulation in tumors with TNM stages III-IV and larger tumors compared with tumors with TNM stage $\mathrm{I}-\mathrm{II}$ and small tumors respectively $(\mathrm{P}-\mathrm{value}=0.0429)$ (Figure 3). Despite non-significant difference, the data showed the potential role of $S N H G 1$ in cancer progression. Moreover, the $S N H G 1$ expression in tumor tissue samples with lymphatic invasion was compared to non-invasive ones that it shows there is a significant increase in gene expression in lymphatic invasive tissue samples $(\mathrm{P}$-value $=0.0054)$ (Figure 3c).

However, this hypothesis needs to be confirmed by more clinical and experimental studies.

\section{Discussion}

Although the incidence and mortality of the colorectal cancer declined for the last decades, it remains one of the most common type of malignant tumors (Siegel et al., 2017). High recurrence rate of CRC urged the identification of novel biomarkers for prognosis and therapies. Recent investigations have revealed that the expression of lncRNAs are dysregulated in a variety of cancers (Huarte et al., 2015; Hajjari et al., 2014; Qiu et al., 2013). Many recent and important discoveries showed a wide involvement of lncRNAs in gene-regulation,

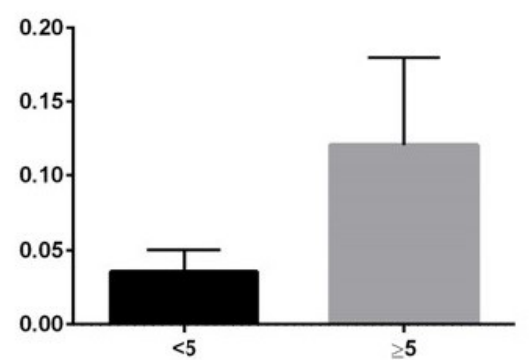

$3 a$

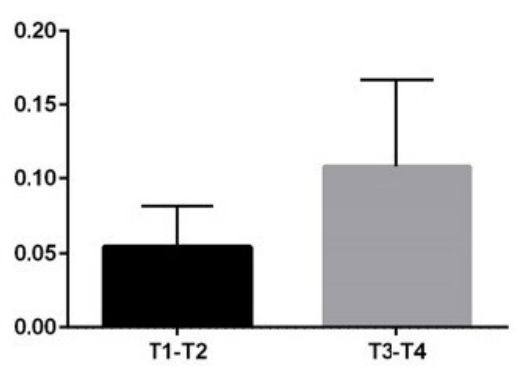

$3 \mathrm{~b}$

Figure 3. Comparison of the SNHG1 Expression between Colorectal Adenocarcinoma Tissues. (a), SNHG1 expression in larger and small tumors (P-value=0.0092); (b), SNHG1 expression in tumors with different TNM stages $(\mathrm{P}$-value $=0.0429)$ 
transport, differentiation, dosage-compensation, and protein synthesis (Chen et al., 2010). The key regulatory function of IncRNAs proposed them as therapeutic targets or biomarkers. So, they could be used a new strategy in cancer early diagnosis. Different studies have reported that the expression of these RNAs are significantly changed in various types of human malignancies especially colon cancer (Gutschner et al., 2012; Prensner et al., 2011).Therefore, exploration of CRC-related lncRNAs and detection of their clinical importance and biological properties may accelerate the development of IncRNA-based diagnostic methods for CRC. Here, we sought the expression of the lncRNA termed small nucleolar RNA host gene1 ( $S N H G 1)$ to find its potential role in colorectal cancer. For this purpose, we focused on the TCGA database and found a significant SNHG1 overexpression in colorectal cancer tissues derived from TCGA large cohort database. Moreover, in order to validate in silico data, we quantified the expression levels of SNHG1 lncRNA by Real-time PCR in tissue samples. Our results for $S N H G 1$ in CRC were consistent with our in silico data. In summary, this study suggests that SNHG1 might play a key potential oncogenic role in colorectal cancer. We also showed that SNHG1 overexpression is potentially associated with advanced TNM stage. Our findings are consistent with the recent study showing the up-regulation of $S N H G 1$ expression in colorectal carcinoma (Sun et al., 2017). However, this is the first study highlighting the up-regulation of SNHG1 in a large cohort of samples validated by experimental analysis on colorectal adenocarcinoma type tissues.

The association of aberrant expression of SNHG1 in human tissues and cell lines with the pathogenesis of different cancers clarifies that it might be implicated in the complex process of tumorigenesis. Researches have shown that overexpression of $S N H G 1$ can promote progression of cancers (Liu et al., 2017; Wan et al., 2016). Recent studies show that $\mathrm{Wnt} / \beta$-catenin signaling pathway is mediated by $S N H G 1$ overexpression in cancers (Cui et al., 2017; Wang et al., 2017). Recently, Sun et al., (2017) found that the expression levels of SNHG1 in human lung and colorectal cancers were higher in patients and the expression level is positively correlated with the promotion of tumor cell growth. Interestingly, this study uncovered that $S N H G 1$ can act in cis and trans through diverse mechanisms to regulate gene expression .Their findings revealed that MYC is a key transcriptional factor that has been regulated in trans. It supports Sahu et al., (2016) finding which indicated that SNHG1 is up-regulated by MYCN amplification in neuroblastoma. Also, SNHG1 promotes the expression of its neighboring gene by cis-acting and induces the formation of chromatin looping of the SNHG1-SLC3A2. So, SNHG1 interferes with the inactivation of the PI3K/AKT pathway by regulating $S L C 3 A 2$ gene (Sun et al., 2017). Sun et al., (2017) and Zhou et al., (2017) examined the expression level of $S N H G 1$ upon knockdown in different cell lines. They showed the importance of SNHG1 expression in CRC. In this study, we studied the expression level of SNHG1 lncRNA to reveal the importance of this gene in CRC patients and this is just as an experimental validation study in our country.

Some other studies on different types of cancers have proposed other mechanisms such as inhibiting apoptosis through P53 target genes (Shen et al., 2017) and TAp63/ZEB1 pathway (Zhang et al., 2017), as well as cell cycle progression through suppression of miR-195 expression (Zhang et al., 2016). This microRNA blocks the $\mathrm{G}(1) / \mathrm{S}$ transition through targeting cyclin D1, CDK6, and E2F3 (Xu et al., 2009). Also, some studies show that $S N H G 1$ may act through regulating of epigenetic state (Hu et al., 2017). Besides that, Tian et al., (2017) and $\mathrm{Xu}$ et al., (2018) reported SNHG1 up-regulated in colorectal cell lines and the differential expression of SNHG1 may indicate the potential role of this lncRNA in cancer initiation and progression. This study was aimed to highlight the potential role of $S N H G 1$ in colorectal cancer. To our knowledge, this is the first experimental validation study highlighting the over-expression of SNHG1 in colorectal cancer.

In conclusion, enhanced $S N H G 1$ expression was observed in colorectal adenocarcinoma tissues. These results indicate that SNHG1 lncRNA is likely to be involved in the development of colorectal cancer. Thus, our data suggest $S N H G 1$ as a potential novel prognostic biomarker or therapeutic target for patients with colorectal adenocarcinoma. However, there is a need to examine this lncRNA a larger scale and long term follow up in order to confirm it. So, the current study can be a basis for further experiments on the exact functions of SNHG1 a potential biomarker or therapeutic target in cancer-related pathways.

\section{Acknowledgments}

We thank Shahid Chamran university of Ahvaz for supporting this study.

\section{Conflict of interest}

None.

\section{References}

Birkenkamp-Demtroder K, Christensen LL, Olesen SH, et al (2002). Gene expression in colorectal cancer. Cancer Res, 62, 4352-63.

Chen LL, Carmichael GG (2010). Decoding the function of nuclear long non-coding RNAs. Curr Opin Cell Biol, 22, 357-364.

Cui Y, Zhang F, Zhu C, et al (2017). Upregulated lncRNA SNHG1 contributes to progression of non-small cell lung cancer through inhibition of miR-101-3p and activation of Wnt/ $\beta$-catenin signaling pathway. Oncotarget, 8, 17785.

Gutschner T, Diederichs S (2012). The hallmarks of cancer: a long non-coding RNA point of view. RNA Biol, 9, 703-19.

Han D, Wang M, Ma N, et al (2015). Long noncoding RNAs: novel players in colorectal cancer. Cancer Lett, 361, 13-21.

Huarte M (2015). The emerging role of lncRNAs in cancer. Nat Med, 21, 1253.

Hajjari M, Khoshnevisan A, Shin YK (2014). Molecular function and regulation of long non-coding RNAs: paradigms with potential roles in cancer. Tumor Biol, 35, 10645-63.

$\mathrm{Hu}$ Y, Ma Z, He Y, et al (2017). LncRNA-SNHG1 contributes to gastric cancer cell proliferation by regulating DNMT1. 
Biochem Biophys Res Commun, 491, 926-31.

Kogo R, Shimamura T, Mimori K, et al (2011). Long noncoding RNA HOTAIR regulates polycomb-dependent chromatin modification and is associated with poor prognosis in colorectal cancers. Cancer Res, 71, 6320-6.

Livak KJ, Schmittgen TD (2001). Analysis of relative gene expression data using real-time quantitative PCR and the $2^{-\triangle \Delta C T}$ method. Methods, 25, 402-8.

Liu Y, Yang Y, Li L (2017). LncRNA SNHG1 enhances cell proliferation, migration and invasion in cervical cancer. Biochem Cell Biol, 96, 38-43

Ponting CP, Oliver PL, Reik W (2009). Evolution and functions of long noncoding RNAs. Cell, 136, 629-41.

Prensner JR, Chinnaiyan AM (2011). The emergence of lncRNAs in cancer biology. Cancer Discov, 1, 391-407.

Qiu MT, Hu JW, Yin R, Xu L (2013). Long noncoding RNA: an emerging paradigm of cancer research. Tumor Biol, 34, 613-20.

Siegel RL, Miller KD, Fedewa SA, et al (2017).Colorectal cancer statistics. CA Cancer J Clin, 67, 177-93.

Sahu D, Hsu CL, Lin CC, et al (2016). Co-expression analysis identifies long noncoding RNA SNHG1 as a novel predictor for event-free survival in neuroblastoma. Oncotarget, 7, 58022.

Sun Y, Wei G, Luo H, et al (2017).The long noncoding RNA SNHG1 promotes tumor growth through regulating transcription of both local and distal genes. Oncogene, 36, 6774-83.

Sun X, Wang Z, Yuan W (2017). Down-regulated long noncoding RNA SNHG1 inhibits tumor genesis of colorectal carcinoma. Cancer Biomarkers, 20, 67-73.

Shen Y, Liu S, Fan J, et al (2017). Nuclear retention of the lncRNA SNHG1 by doxorubicin attenuates hnRNPC-p53 protein interactions. EMBO Rep, 6, e201643139.

Torre LA, Bray F, Siegel RL, et al (2012). Global cancer statistics. CA Cancer J Clin, 65, 87-108.

Tian T, Qiu R, Qiu X (2018). SNHG1 promotes cell proliferation by acting as a sponge of miR-145 in colorectal cancer. Oncotarget, 9, 2128.

Wang KC, Chang HY (2011). Molecular mechanisms of long noncoding RNAs. Mol Cell, 43, 904-14.

Wang Q, Li Q, Zhou P, et al (2017).Upregulation of the long non-coding RNA SNHG1 predicts poor prognosis, promotes cell proliferation and invasion, and reduces apoptosis in glioma. Biomed Pharmacother, 91, 906-11.

Wan X, Huang W, Yang S (2016). Identification of androgenresponsive lncRNAs as diagnostic and prognostic markers for prostate cancer. Oncotarget, 7, 60503.

Wang S, Jiang J, Wang Z, Xie Y, Wu X (2017). Long non-coding RNA SNHG1 is an unfavorable prognostic factor and promotes cell proliferation and migration by $\mathrm{Wnt} / \beta$-catenin pathway in epithelial ovarian cancer. Int J Clin Exp Pathol, 10, 9284-92.

Xie X, Tang B, Xiao YF, et al (2016). Long non-coding RNAs in colorectal cancer. Oncotarget, 7, 5226.

Xu MD, Qi P, Du X (2014). Long non-coding RNAs in colorectal cancer: implications for pathogenesis and clinical application. Mod Pathol, 27, 1310.

Xu T, Zhu Y, Xiong Y, et al (2009). MicroRNA-195 suppresses tumorigenicity and regulates G1/S transition of human hepatocellular carcinoma cells. Hepatology, 50,113-21.

$\mathrm{Xu}$ M, Chen X, Lin K, et al (2018). The long noncoding RNA SNHG1 regulates colorectal cancer cell growth through interactions with EZH2 and miR-154-5p. Mol Cancer, 17, 141.

You J, Fang N, Gu J, et al (2014) .Noncoding RNA small nucleolar RNA host gene 1 promote cell proliferation in nonsmall cell lung cancer. Indian J Cancer, 51, 99.

Zhang HY, Yang W, Zheng FS, et al (2017). Long non-coding RNA SNHG1 regulates zinc finger E-box binding homeobox 1 expression by interacting with TAp63 and promotes cell metastasis and invasion in Lung squamous cell carcinoma. Biomed Pharmacother, 1, 650-8.

Zhu Y, Li B, Liu Z, et al (2017). Up-regulation of IncRNA SNHG1 indicates poor prognosis and promotes cell proliferation and metastasis of colorectal cancer by activation of the Wnt/ $\beta$-catenin signaling pathway. Oncotarget, 8, 111715.

Zhang M, Wang W, Li T, et al (2016). Long noncoding RNA SNHG1 predicts a poor prognosis and promotes hepatocellular carcinoma tumorigenesis. Biomed Pharmacother, 1, 73-9.

Zhang H, Zhu D, Ying M, et al (2016). Expression of long non-coding RNA (lncRNA) small nucleolar RNA host gene 1 (SNHG1) exacerbates hepatocellular carcinoma through suppressing miR-195. Med Sci Monit, 22, 4820.

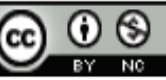

This work is licensed under a Creative Commons AttributionNon Commercial 4.0 International License. 